\title{
Granules in Granular Cell Lesions of the Head and Neck: A Review
}

\author{
T. Lakkashetty Yogesh ${ }^{1}$ and S. V. Sowmya ${ }^{2}$ \\ ${ }^{1}$ Department of Oral Pathology and Microbiology, Sri Rajiv Gandhi College of Dental Sciences, Bangalore, \\ Karnataka 560032, India \\ ${ }^{2}$ Sri Rajiv Gandhi College of Dental Sciences, Cholanagar, Hebbal, R.T. Nagar post, Karnataka 560032, Bangalore, India
}

Correspondence should be addressed to T. Lakkashetty Yogesh, yogesh50181@rediffmail.com

Received 9 May 2011; Accepted 11 June 2011

Academic Editors: G. Acs and A. B. Galosi

Copyright ( 12011 T. L. Yogesh and S. V. Sowmya. This is an open access article distributed under the Creative Commons Attribution License, which permits unrestricted use, distribution, and reproduction in any medium, provided the original work is properly cited.

\begin{abstract}
Granular cell lesions of the oral mucosa, jaws, and salivary glands constitute a heterogeneous group of lesions which may be either odontogenic, salivary gland, or metastatic in origin. Granular cells in these proliferations most commonly are the result of lysosomal accumulation, aging, degenerative, metabolic alteration, increased apoptosis, cytoplasmic autophagocytosis, and many more. Many benign and malignant tumors that occur in the oral cavity contain granular cells as a characteristic component of their pathology. Sometimes dilemma exists in the proper diagnosis of these granular cell lesions and the cell of origin because they share similar light and electron microscopic features. Therefore, immunohistochemistry helps to confirm histologic impressions and differentiate other neoplastic entities with granular cytoplasmic features. Granularity in a normal histopathology is a rare but innocuous change and does not influence the biologic behaviour of smooth muscle tumors except few lesions such as cutaneous granular cell angiosarcoma and granular cell ameloblastoma which have shown poor prognosis. This paper aims to review the clinical and pathologic features, different immunohistochemical profiles of granules in granular cell lesions of head and neck with an attempted working classification.
\end{abstract}

\section{Introduction}

Tumours and tumour-like lesions of the oral cavity composed predominantly of granular cells are uncommon. The nature of various oral granular cell lesions is unclear, and many theories have been proposed for the origin of granules, the principal ones are odontogenic, fibroblastic, histiocytic, myoblastic, and neurogenic. Granular cells are also seen associated with the enamel organ of developing tooth [1].

These granular cell neoplasms represent a heterogenous group including epithelial and mesenchymal tumours. Therefore, immunohistochemistry and electron microscopy help to confirm histologic impression and differentiate other neoplastic entities with granular cytoplasmic features [2]. The morphology of all granular cells is similar, but their origins are different [3]. Because of their common yet inexplicable morphologic appearance and obscure origin, granular cell lesions continue to be a focus of laboratory investigation, and many investigations are done to know the antigenic heterogeneity of granular cell lesions from a wide variety of sites in skin, mucosa, and jaw [4].

\section{Classification}

Review of the literature has shown that only few authors have attempted to classify granular cell lesions. One such classification on oral granular cell lesions including odontogenic and nonodontogenic tumors was proposed, but has not included many granular cell lesions [5]. Hence we made an attempt to classify granular cell lesions as below.

(I) Odontogenic:

(1) granular cell ameloblastoma,

(2) central and peripheral granular cell odontogenic tumor/granular cell Ameloblastic fibro$\mathrm{ma} /$ central granular cell odontogenic fibroma,

(3) calcifying epithelial odontogenic tumor,

(4) granular cell odontogenic cyst (variant of lateral periodontal cyst). 
(II) Nonodontogenic neoplasms:

(A) Soft tissue neoplasms:

(a) Benign:

(1) granular cell tumor,

(2) congenital epulis of newborn,

(3) leiomyoma,

(4) rhabdomyoma,

(5) neurilemmoma,

(6) granular cell traumatic neuroma,

(7) paraganglioma,

(8) dermatofibroma,

(9) perivascular epithelioid cell tumor.

(b) Malignant:

(1) alveolar soft part sarcoma,

(2) malignant granular cell tumor,

(3) rhabdomyosarcoma,

(4) hodgkin's lymphoma,

(5) basal cell carcinoma,

(6) angiosarcoma,

(7) dermatofibrosarcoma.

(B) Salivary gland diseases (oncocytic lesions):

(a) Benign neoplasms:

(1) Warthin's tumor,

(2) oncocytoma,

(3) canalicular adenoma.

(b) Malignant neoplasms:

(1) acinic cell carcinoma,

(2) mucoepidermoid carcinoma,

(3) epithelial-myoepithelial carcinoma,

(4) oncocytic carcinoma,

(5) salivary duct carcinoma.

(c) Nonneoplastic lesions:

(1) sialadenosis,

(2) oncocytosis.

(C) Melanocytic lesions:

(1) pigmented basal cell carcinoma,

(2) malignant melanoma,

(3) naevus,

(4) melanocytic macule.

(D) Histiocytic lesions:

(1) Langerhans cell histiocytosis,

(2) verruciform xanthoma,

(3) xanthogranuloma,

(4) atypical fibroxanthoma.

(E) Reactive lesions:

(1) gingival hyperplasia,

(2) traumatic sites,

(F) Metastatic lesions:

(1) granular cell variant of renal cell carcinoma,

(2) metastasis from carcinoma of breast,

(3) metastatic malignant melanoma,

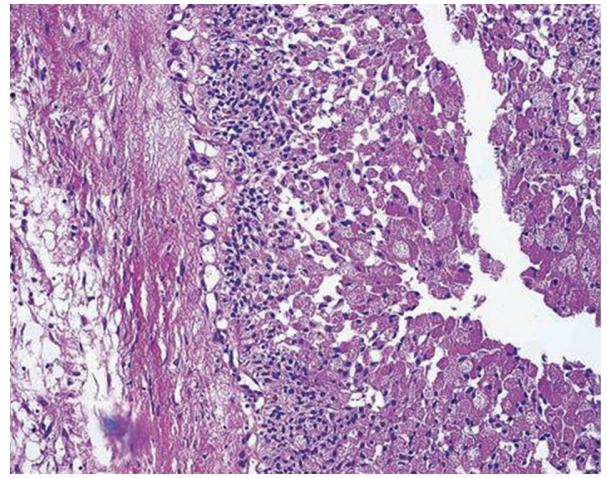

Figure 1: Photomicrograph of granular cell ameloblastoma with prominent granularity of the central cells (hematoxylin and eosin stain, $\times 100)$ (courtesy: http://www.pathconsultddx.com/).

(4) metastatic oncocytic carcinoma of thyroid,

(5) metastatic hepatocellular carcinoma,

(G) Miscellaneous:

(1) fibrous papule of the nose,

(2) granular cell lichen planus.

\section{Granular Cell Ameloblastoma (GCA)}

GCA is known to be aggressive histologic variant among all the ameloblastoma (Figure 1). It is important to separate GCA from other ameloblastomas because of a higher incidence of malignancy and metastases $[6,7]$. The granular appearance has been ascribed to numerous lysosomes based on histochemical and electron microscopic findings. Ultrastructurally, the osmiophilic internal structure of the lysosomes varies considerably. Many of these granules approach $1 \mu \mathrm{m}$ in size; giant granules of $30 \mu \mathrm{m}$ in diameter are rarely seen. They present with features of finger-printlike membranous structures, myelin figures, small particles, granules, vesicles, lattice structures, and crystalloids. This diversity may represent different materials and stages of digestion of the lysosomal contents. The myelin figures suggest the presence of phospholipid in the granules. Therefore, it has been concluded by many authors that numerous lysosomes represent increased cellular actions of the tumour ameloblasts to digest unwanted components [3]. During normal amelogenesis, an increase in the autophagic lysosomes has been observed in the ameloblasts between secretory and absorptive stages and from reduced ameloblasts to squamous epithelium. Accumulation of granular cells is also noted in enamel organ of deciduous tooth [1]. This may be due to lysosomal insufficiency or overproduction of unused material in the odontogenic epithelium. Thus, odontogenic epithelium seems to be able to undergo granular change under certain conditions [3].

Several theories have been suggested to explain the granular change including aging or degenerating process. It is speculated that unnecessary or aged components in the cytoplasm of some tumour cells become increasingly more numerous with age; however, the ability of lysosomes to 
digest or dispose these materials decreases with age, and as a result the cytoplasm of some tumour cells become packed with lysosomal granules [3]. On immunohistochemical characterization of cellular differentiation, granular cells are positive for cytokeratin, CD68, lysozyme, and alpha1 -antichymotrypsin, but negative for acid phosphatase, $\alpha$ naphthyl acetate, $\beta$-glucuronidase, vimentin, desmin, $\mathrm{S}$ 100 protein, neuron-specific enolase, and CD15, indicating epithelial origin and lysosomal aggregation. These features suggest that the cytoplasmic granularity in GCA might be caused by increased apoptosis and associated phagocytosis by neighbouring neoplastic cells $[3,8]$.

\section{Central Granular Cell Odontogenic Tumour (CGCOT)}

It is also known as central granular cell odontogenic fibroma or granular cell ameloblastic fibroma which is an exceedingly rare benign odontogenic neoplasm containing varying amount of large eosinophilic granular cells and apparently inactive odontogenic epithelium. Many GCAs may indeed represent CGCOT [9]. Histogenesis of CGCOT remains controversial, and many authors suggested that CGCOT may be a later manifestation of the same disorder which leads to the formation of congenital epulis of the newborn; alteration in the mesenchymal component represents an ageing or degenerative response in the ameloblastic fibroma, origin from fibroblasts, and was identical to the cells in the granular cell myoblastoma. It is also postulated that residual ectomesenchymal influence may be responsible for proliferation of both gingival epithelium and mesenchymal cells which are subsequently transformed into granular cells $[10,11]$. Kumamoto and Ooya reported that granular cells are of epithelial origin and the result of processes of an involuted and dystrophic nature [8]. The granular cells are positive for vimentin, CD68, lysozyme, muscle-specific actin, alpha-smooth muscle actin, calponin, neuron-specific enolase (NSE), CD138, and bcl-2 [12].

Granular cells are arranged in sheets or lobules which are ovoid to round in shape, varying from 20 to $50 \mu \mathrm{m}$ with abundant eosinophilic granular cytoplasm and eccentric round to oval nuclei $[9,10]$. Granular cell cytoplasm is Sudan black B and periodic acid Schiff (PAS) positive, diastase resistant, and negative for mucicarmine stain $[11,13,14]$. Ultrastructural findings reveals that all granular cells contain numerous lysosome-like granules, with little cytoplasmic organelles and markedly indented nuclei. The lysosome-like granules of granular cells show the following appearances: electron-dense and amorphous granules, aggregates of fine granular particles, vesicular granules, granules with myelinlike structure, and granules with mixed appearance of them [10]. Many authors have shown ultrastructurally numerous lysosome-like bodies; Type I membrane-bound dense body composed of an electron-dense homogeneous material, Type II aggregate of virus-like particles bound by membrane measuring up to $300 \AA$ in diameter, Type III large structure composed of the first two types and bound by a membrane. This type is more common than the first and second types.

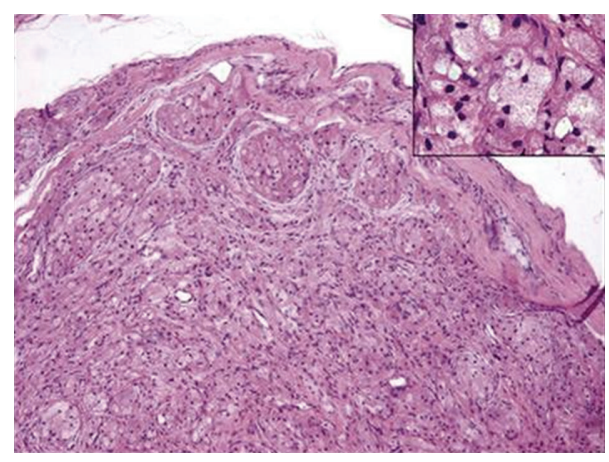

FIGURE 2: Photomicrograph showing histopathology of granular cell tumor. Lesion of the lip showing large polyhedral neoplastic cells with eosinophilic granular cytoplasm and small vesicular or hyperchromatic nuclei separated by fibrous collagen, and the inset shows the granular cell component (hematoxylin and eosin stain, $\times 125)[18]$.

Type I and Type III are believed to represent lysosomes whereas; Type III alone is thought not to represent lysosome, and its function is not known [15].

\section{Calcifying Epithelial Odontogenic Tumour (CEOT)}

CEOT accounts for $1 \%$ of all benign odontogenic tumors and is of epithelial origin. It characteristically contains calcified masses and homogenous acellular material within the tumor epithelium and stroma. Most polyhedral granular cells in CEOT are PAS-negative; however, few cells contain PAS positive granular material which can be removed by reaction with diastase. A great deal of cytoplasmic density appears to be due to polyribosomes, secondary lysosomal bodies of the autophagic variety. Many of these structures are of dense body type, but, in addition, other morphological variants of lysosomes such as autophagic vacuoles, myelin figures, and multivesicular bodies are observed [16].

\section{Granular Cell Odontogenic Cyst}

Granular cell odontogenic cyst is a unicystic (monocystic) granular cellameloblastoma, and the presence of granular cells in ameloblastoma is not a permanent feature and may be of little value as a prognostic indicator of aggressiveness. It is considered to be a variant of unicystic ameloblastoma of granular cell type and should be treated as such, and the granular cell component cannot be used as prognostic criteria [17].

\section{Granular Cell Tumor (GCT)}

This tumour is also termed as tumour of Abrikosoff, granular cell myoblastoma, granular cell neurofibroma, or granular cell schwannoma (Figure 2). The most frequent orofacial localization is tongue. New hypothesis based on immunohistochemical studies were developed implicating 


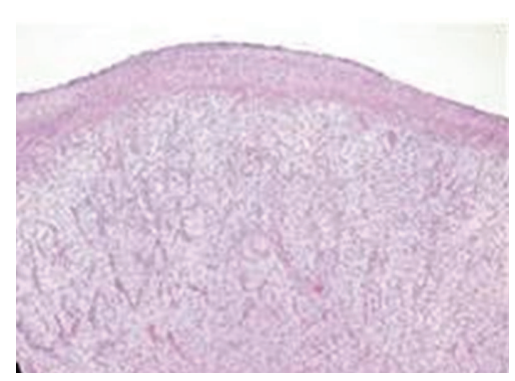

(a)

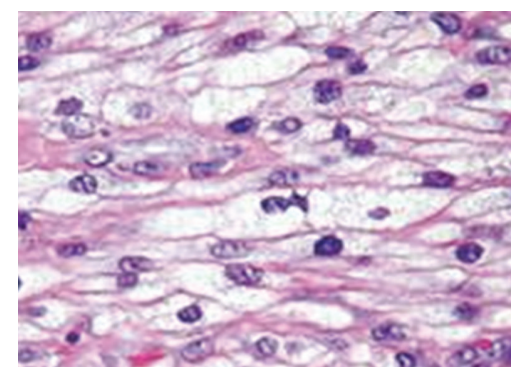

(c)

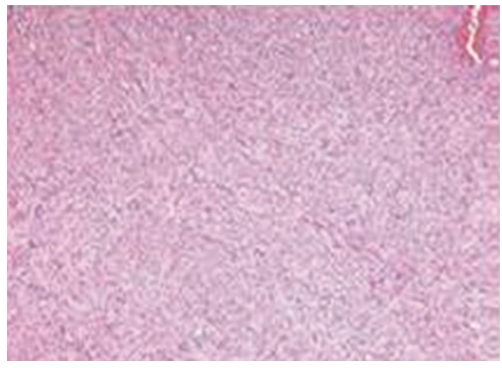

(b)

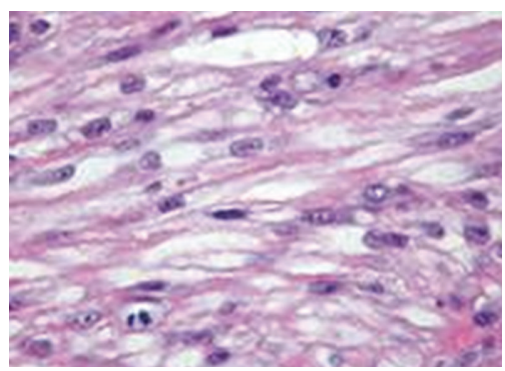

(d)

FIGURE 3: Photomicrograph showing histopathology of primitive polypoid granular cell tumour (PPGCT). (a) Ulcerated mucosa overlying a tumor composed of sheets of granular cells and clear cells (hematoxylin-eosin stain, $\times 40$ ). (b) Spindle cells and scattered clear cells arranged in fascicles (hematoxylin-eosin stain, $\times 40$ ). (c) Spindle cells with granular cytoplasm (hematoxylin-eosin stain, $\times 40$ ). (d) Spindle cells with granular and clear cytoplasm; mitotic figures are seen (hematoxylin-eosin stain, $\times 400$ ) [19].

mesenchymal cells, neural crest cells, histiocytes, or Schwann cells in the histogenesis of GCT [20]. Presence of autophagic vacuoles and positive IHC staining for $\alpha-1$ antichymotrypsin and $\alpha-1$ anti-trypsin suggest histiocytic origin [21].

At present, most of the authors consider that the Schwann cells are the precursors of this tumor because of positive staining for S-100, vimentin, glycoprotein or leu7 , neurofilament, NSE, neurotubules and the presence of structures compatible with myelin inside the liposomes [20]. Positive staining for Luxol fast blue, a myelin stain supports the neural origin for GCT [19]. Granular cytoplasmic change is the expression of metabolic change occurring in Schwann's cell tumours [22].

Granular cells have in common the presence of lysosomes and generally stain for NK1/C3 (CD 63) (NK1/C3 is a nonspecific lysosomal membrane glycoprotein), a neuroectodermal and lysosomal marker that stains cells in the tumour of neural crest origin such as melanoma and nevomelanocytic nevi. KP1 (CD 68), another lysosomal marker, has also shown positivity in GCT $[19,23]$. All these suggest presence of lysosomes within the cytoplasm of granular cells. Granular cells can occur as a degenerative, reactive, or hamartomatous phenomenon [19]. Granules are also PAS positive; contain glycoprotein, lipoid material and polysaccharides [24].

Nonneural GCTs of the oral cavity which was originally described under the designation, primitive polypoid granular cell tumour (PPGCT) (Figure 3), have been reported which differ from traditional GCTs in that they show expansile growth, cellular atypia, mitotic activity, and negative staining for S-100 and NSE [19, 23]. Granular cell changes have been reported in sites of previous surgical trauma and shows features of both GCT and traumatic neuroma. Many such tumors are reported in salivary glands and have been designated as granular cell traumatic neuroma [25]. The granular change inside the nerve bundle is similar to the Wallerian degeneration, and the cytoplasmic granularity is probably derived from lysosomal engulfment with myelin products as in GCT [26].

\section{Congenital Epulis of Newborn (CEN)}

CEN is also called congenital granular cell lesion (CGCL), congenital epulis, Neumann's tumour, or congenital gingival granular cell tumor [27]. Exact etiology of CEN is not known, but numerous theories have been postulated including odontogenic, fibroblastic, histiocytic, myogenic, and neurogenic. Electron microscopic and immunohistochemical studies have shown origin from other tissues like epithelial, undifferentiated mesenchymal cells, pericytes, smooth muscle cells, and myofibroblasts [27]. IHC findings have suggested that lesional cells are derived from neural crests but do not show any significant neural derivation. Cells of the lesion represent early mesodermal cells that express pericytes or myofibroblastic features that undergo cytoplasmic autophagocytosis at a later stage. There is a lot of similarity histologically between CGCL and GCT. IHC studies have demonstrated some difference, including absence of S-100, $75 \mathrm{kD}$ nerve growth receptor factor, trk gene product, and phosphotyrosine-positive cells in CGCL but their presence in GCT [28]. 


\section{Granular Cell Leiomyoma}

Leiomyomas are rare, which accounts for $0.42 \%$ of all soft tissue lesions of the oral cavity, and arise from the smooth muscle of the vasculature. It is classified as solid, angiomyoma and epithelioid variants. Smooth muscle tumors demonstrate a number of histopathological alterations including epithelioid, clear cell, granular cell, and myxoid change. Granular cell change in leiomyoma is an infrequently reported alteration.

The tumor usually shows large polygonal to fusiformshaped cells with abundant eosinophilic, granular cytoplasm; the nuclei are round to ovoid, hyperchromatic with absence of mitosis (Figure 4). Granular appearance is thought to be a degenerative change, and it may be due to tissue response to various factors including neoplasia, degenerative process, and reactive process such as anoxia, sublethal injury of the cells due to excessive proliferation of surrounding collagenous fibers, abnormal increase in the lysosomes or lipids, metabolic storage disorders, and defective lysosomes [29, 30]. Granular cells in leiomyoma also demonstrate positive staining for $\alpha$ - 1 antitrypsin and PAS with diastase resistance.

Granular cell change in leiomyoma may easily be confused with GCT, but typical spindle cell component is absent in GCT which helps the in differentiation. Positive immunohistochemistry for smooth muscle actin (SMA), desmin and negative staining for S-100 supports smooth muscle origin compared to GCT. Another differential diagnosis includes granular cell schwannoma, but lack of reactivity to S-100 and positive staining for SMA and HHF-35 favours smooth muscle tumour. Perivascular epitheloid cell tumor and granular cell variant of clear cell sarcoma are also in the differential diagnosis of leiomyoma. The former shows positivity for premelanosomes, melanocytic markers and negative for S-100; whereas the latter is positive for S-100 and HMB-45. Granular cell change does not appear to influence the biologic behaviour of these lesions [29].

\section{Perivascular Epithelioid Cell Tumor (PEComas)}

PEComas are family of tumors defined by the coexpression of melanocytic and muscle markers. It most commonly occurs in pancreas, bladder, prostate, thigh, nasal cavity, abdomen, breast, and very rare in oral cavity. The majority of tumors composed of PEComas occur in patients with tuberous sclerosis complex (TSC). But PEComa without TSC can occur which results from postzygotic somatic mutations in the same genes. Histologically, PEComas show large elongated or epithelioid cells with granular eosinophilic cytoplasm with occasional cytoplasmic clearing, centrally located nuclei, occasional nuclear hyperchromatism, limited collagenous component, minute capillaries, and occasional thick-walled capillaries [31].

PEComas are of interest primarily because of their immunohistochemical reaction with melanocytic and muscle-specific markers such as HMB-45, Melan-A/MART1, microphthalmia transcription factor, and CD10 and almost

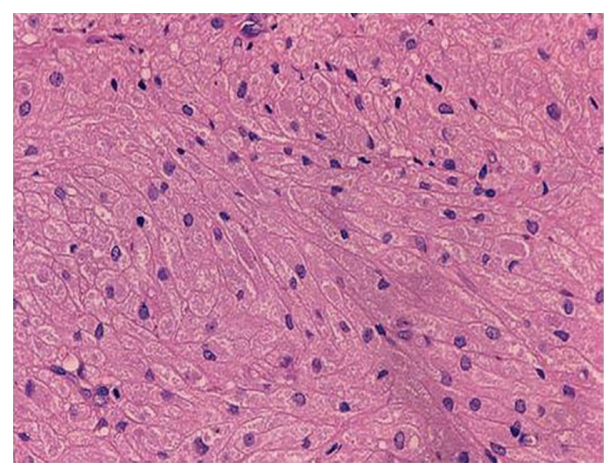

FIgURE 4: Photomicrograph showing histopathology of granular cell leiomyoma. The tumor is primarily composed of large polygonal to fusiform-shaped cells with abundant, eosinophilic, granular cytoplasm. The nuclei are round to ovoid and hyperchromatic (hematoxylin and eosin stain, $\times 400$ ) [27].

negative for S-100. Elongated cells in PEComas show positivity for SMA, desmin, and muscle-specific actin $[23,31]$. Two hypotheses are proposed to explain the histogenesis of PEComas. One hypothesis is that PEComas are derived from undifferentiated cells of neural crest that can express dual smooth muscle and melanocytic phenotype. A second hypothesis is that these tumors are of myoblastic and more specifically smooth muscle origin but are molecularly altered and coexpress melanogenesis and melanocytic markers. The differential diagnosis of PEComa includes GCT, rhabdomyoma, clear cell sarcoma of the soft parts, alveolar soft part sarcoma, epithelioid leiomyoma, and melanoma [31].

\section{Granular Cell Basal Cell Carcinoma (GBCC)}

Basal cell carcinoma (BCC) is a cutaneous tumor which occurs in a number of histological forms. Granular cell BCC was first described by Barr and Graham. GBCC is seen more in men, majority occur in the face. Histologically GBCC shows well-demarcated tumor mass in nodules which come in contact with basal cell layer of the epidermis and invade the dermis. Tumor cells show pleomorphic lysosomelike granules with eosinophilic cytoplasm and peripheral palisading arrangement of the basal cells. Cytoplasmic granules are PAS positive, 0.1-0.5 $\mu$ lamellar lysosome-like structures similar to GCT. Immunohistochemistry (IHC) do not express S-100, epithelial membrane antigen (EMA), carcinoembryonic antigen (CEA) but express most epithelial antigens such as keratins, Ber-P4 antigen, bcl-2, and p63 proteins. The granules are also positive for CD15 and CD68 which suggests lysosomal nature [32]. The granular material is also interpreted as dense pinocytotic granules, collagenase zymogen granules, and acid phosphate positive which develop as a result of degenerative process. Certain studies have shown that mitochondrial proliferation is attributed to a compensatory hypertrophy mechanism operating at the subcellular level in response to defective mitochondrial function contributing to its granularity [33]. 


\section{Alveolar Soft Part Sarcoma (ASPS)}

It is a rare tumour which occurs mostly in young adults with a highly characteristic histopathology, ultrastructure, controversial histogenesis, and often enigmatic clinical behaviour [34]. It usually involves muscles and deep soft tissues of the extremities. In head and neck, tongue and orbit are the most common sites. ASPS was previously published under a variety of different names including malignant myoblastoma, granular cell myoblastoma, and a malignant GCT [35]. The favoured possibilities for the derivation of ASPS are neural or neuroendocrine, myogenic, and paraganglion differentiation.

Lesional granular cells have distinct borders and abundant eosinophilic granular cytoplasm resulting in epithelioid appearance, round, regular eccentrically placed nucleus, vesicular chromatin, prominent nucleoli with infrequent mitosis, and necrosis (Figure 5). ASPS cells are negative for epithelial markers such as cytokeratin, epithelial membrane antigen (EMA), S-100, neuroendocrine markers such as chromogranin-A, synaptophysin, and melanocytic markers such as HMB-45 and melan-A. ASPS cell granules and crystals contain protein and polysaccharides which can be identified with Alcian blue, trichome, and PAS histochemical stains. Ultrastructure shows crystalline structures which may be rhomboid, rod-like, or spiked consisting of aggregates of monocarboxylate transporter protein (MCT1) and its cellular chaperone CD147 [33].

\section{Paraganglioma}

Paragangliomas are neuroendocrine tumors arising from extra-adrenal autonomic ganglia. Head and neck paraganglia occur in association with the carotid body, the ganglion nodosum of the vagus nerve, the middle ear (jugulotympanic paraganglia), and in other rarer sites where paraganglia are known to exist. The lesions are usually composed of round nests of uniform polygonal chief cells with extensive granular eosinophilic cytoplasm. The nuclei of these neoplastic cells are large, regular, centrally located, and shows occasional vesiculation with no mitoses. Nests of polygonal chief cells are separated by the cytoplasmic processes of elongated sustentacular cells, associated with fibrovascular stroma. There will be no significant gland formation, anaplasia, necrosis, mitotic activity, or invasion in the biopsy specimens. Electron microscopy reveals a chief cell ultrastructure that is highlighted both by large numbers of mitochondria and, particularly, by numerous 100 - to 200-nm, uniform neurosecretory granules. Grimelius and chromogranin A stains confirm the neurosecretory nature of the chief cell granules and together with neuron-specific enolase establish that the tumor arises from a neuroendocrine cell lineage, rather than a modified muscle cell type [36].

\section{Dermatofibroma (DF)}

Dermatofibroma is a common benign fibrohistiocytic cutaneous lesion, most common in the extremities, rare in the oral and perioral region [37].

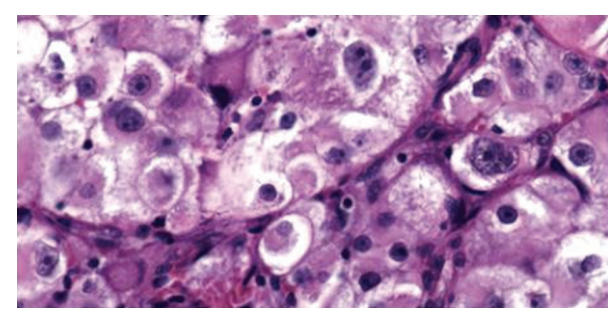

FIGURE 5: Photomicrograph showing histopathology of alveolar soft part sarcoma with relative cellular uniformity and granular cytoplasm and peripherally placed vesicular nuclei containing a prominent central nucleolus (hematoxylin and eosin stain, $\times 400$ ) [33].

An unusual variant of dermatofibroma (DF) in which areas of granular cells are a prominent feature has been reported, and the diagnosis of DF was confirmed by immunohistochemistry and by ultrastructural studies. Cellular granularity is a nonspecific phenomenon characterized by intracytoplasmic accumulation of lysosomes and may cause diagnostic difficulties. Traumatic factors may be involved in the pathogenesis of cellular granularity [38].

\section{Rhabdomyoma}

The adult type is more common and occurs mainly in the head and neck of adult men. Characteristically, it has a welldefined margin and is composed of sheets of closely packed large round cells with granular eosinophilic cytoplasm, some containing cross striations. Cells are positive for musclespecific actin, desmin, and myoglobin (100\%), PAS and diastase sensitive (detects glycogen), PTAH and Masson trichrome which highlight cross striations and rod-like inclusions [39].

\section{Reactive Granular Cells at the Sites of Trauma}

Peculiar, coarsely granular cells, superficially resembling those of myoblastoma and thought to be histiocytic (reactive), were described by Hamperl and others. These cells are frequently found near smooth muscle in sites of previous trauma. The granules are PAS, acidphosphatase, neutral and acid mucopolysaccharide-positive, and contain acid fast, fluorescent, yellow-brown pigment. The granular cells with few cytoplasmic organelles were felt to be an end stage of the undifferentiated cell following incorporation of large quantities of cellular debris. The histiocytic cell granules resemble the so-called "Hamazaki-Wesenberg" $(\mathrm{H}-$ W) bodies, which also contain a lipofuscin- or ceroid-like pigment. Cellular granularity is well described in smooth muscle lesion that range from reactive changes to benign and malignant neoplasms [40]. A case of lichen planus and reactive gingival hyperplasia with rare granular cell change in bilateral buccal mucosa in cells of lamina propria and also have been reported, but their nature and histogenesis has not been revealed [41]. 


\section{Oncocytic Lesions of Salivary Glands}

Hamperl is considered to be the "father" of oncocytes, originally referred to as "onkocytes." He chose this word because of the Greek word "onkousthai," which means "increase in bulk," swollen, enlarged, or tumor. Hamperl described oncocytes in many organs, including parotid, submaxillary, sublingual and minor salivary glands, the thyroid, parathyroid, pituitary gland, adrenal gland, gallbladder, uterus, testicle, fallopian tube, pancreas, liver, stomach, kidney, lung, pharynx, trachea, and esophagus; more recent publications reported oncocytes in the ovary, lacrimal caruncle, breast, and thymus. It is generally accepted that, for a tumor to be called an oncocytoma, it must be comprised exclusively of oncocytes.

Oncocytes were originally thought to represent a degenerative or senescent process, especially because oncocytes can be observed in otherwise normal specimens from aging patients. It appears more likely that they represent redifferentiation of cells with an increased, unbalanced metabolism trying to increase the output of high-energy phosphate. Because mitochondria are somewhat independent organelles, another theory proposes that oncocytomas may represent a neoplasm of subcellular organelle [44].

IHC using antimitochondrial antibody proves to be highly sensitive and specific method for light microscopic identification of mitochondria and superior to routine H\&E or PTAH $[45,46]$.

Oncocytomas are benign salivary gland neoplasms that represent approximately $1.5 \%$ of all salivary gland tumors. They are comprised of oncocytes and generally involve the parotid gland. Histologically, the tumor cells are arranged in solid sheets, alveoli, nests, columns, or cords that are separated by a delicate fibrovascular network. Pseudoacinar arrangement with a small amount of debris in the "lumen" can also be seen. The cells are polyhedral to round with distinct cell borders. Abundant cytoplasm containing varying number of fine to coarse and eosinophilic granules are seen (Figure 6) [44].

Acinic cell carcinoma can be differentiated from oncocytic carcinoma by the presence of amphophilic or basophilic granules in the cytoplasm of tumor cells and their prominent microcystic and papillary growth pattern. IHC shows positivity for mitochondrial antigen, keratin, and alfa-1antichymotrypsin. Primary oncocytic carcinoma of salivary gland (Figure 7) should be differentiated from metastatic oncocytic tumors in salivary glands by the precise clinical and IHC studies [46].

Salivary oncocytic mucoepidermoid carcinoma $(M E C)$ is a very rare subtype of malignant neoplasm, and the granularity is due to the presence of numerous mitochondria which is immunohistochemically positive for mitochondrial antigen and $\alpha 1$ anti-chymotrypsin [47]. Nuclear and cytoplasmic granularity is also noted in the duct-like cells of epithelialmyoepithelial carcinoma. The cytoplasmic granules which are PAS positive are glycogen storage granules. Ultrastructurally numerous mitochondria and other organelles with electron dense secretory granules are seen [48].

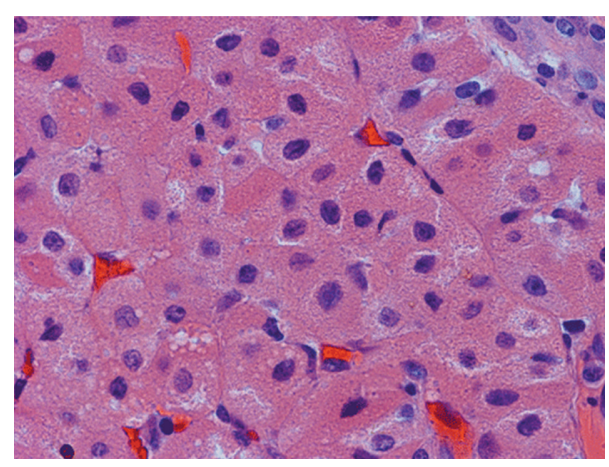

FIGURE 6: Photomicrograph of oncocytoma showing finely granular and distinctly eosinophilic cytoplasm. (hematoxylin and eosin stain, $\times 400)[42]$.

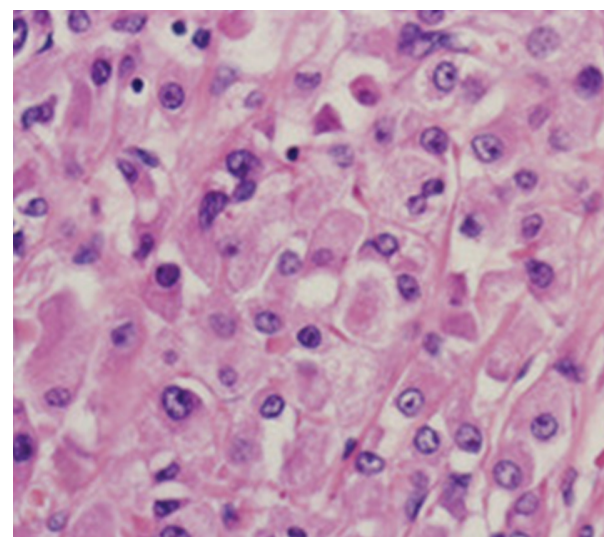

FIGURE 7: Photomicrograph of oncocytic carcinoma showing neoplastic elements with abundant granular eosinophilic cytoplasm, large nuclei, and evident nucleoli, which are large, round, or polyhedral cells arranged in solid sheets, islands, and cords (haematoxylineosin, $\times 400)($ http://www.wjso.com/content/4/1/54).

Metastatic oncocytic carcinoma of thyroid (Hurthle cell carcinoma) can be differentiated by the IHC's expression of thyroglobulin. Metastatic granular variant of renal cell carcinoma (Figure 8) is differentiated by the tumour cells arranged in sheets, organoid nests, cords, or as papillary fronds and are positive for carcinoembryonic antigen and S100 protein $[46,49]$.

Metastatic breast carcinoma may assume many patterns. Cutaneous, pulmonary, and lymph node metastases of an adenocarcinoma of the breast that resembles granular cell tumor has been reported. In all sites, the lesion stained positive with antibodies against EMA and cytokeratins but failed to stain with anti-S100 [50].

Metastatic hepatocellular carcinoma (HCC) sometimes presents as a gingival mass, and the lesion shows large cells arranged in trabecular pattern, abundant eosinophilic granular cytoplasm, pleomorphic nuclei, and prominent nucleoli. IHC with alpha fetoprotein, $\alpha-1$ antichymotrypsin, and Hep-Par-1 help in distinguishing metastatic HCC from other oral metastatic tumors of unknown primary [51].

17.1. Juvenile Xanthogranuloma (JXG). It is a nonLangerhans cell histiocytosis, which is reactive rather 


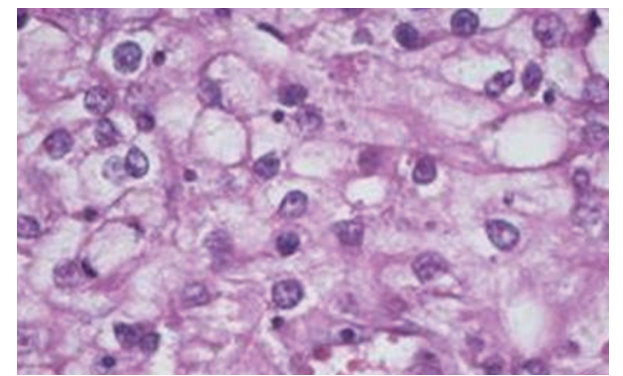

FIgURe 8: Photomicrograph of granular cell variant of renal cell carcinoma (hematoxylin and eosin stain, $\times 400$ ) [43].

than neoplastic process. Accurate diagnosis of oral JXG depends on microscopic and IHC staining. The main cell is the mononuclear histiocyte expressed in 5 different ways: vacuolated, spindle, xanthomatized, scalloped, and oncocytic. Oncocytic cell type of JXG reveals many cells with a rather pronounced, homogenous, slightly granular cytoplasm, and fairly well-demarcated cell borders, with huge multinucleated cells with ground glass appearance of the cytoplasm [52]. JXG can be differentiated from Langerhans cell histiocytosis by electron microscopy, which contains Birbeck's granules that have a pentalaminar, rodlike, tubular structures with characteristic periodicity and occasional dilated terminal ends [53].

17.2. Cutaneous Granular Cell Angiosarcoma (CGCA). Cutaneous angiosarcoma occurring in the head of an elderly person is a well-known entity, which displays typical architectural features of irregular anastomosing vascular channels lined by variably pleomorphic endothelial cells with hyperchromatic nuclei in the dermis and subcutis. CGCA is a rare variant which shows granular eosinophilic cytoplasm and are positive for UEA1 (lectin), CD34, 31, 68. CGCA patients have short survival rate compared to regular cutaneous angiosarcoma [54].

\section{Conclusion}

There are various benign and malignant lesions that may display granular cell features. Granularity in an otherwise normal histopathology from suspected usual granular cell lesions such as GCT, GCA, ASPS, and CEN makes no surprise during the diagnosis. But rare granular change as seen in other lesions such as leiomyoma, BCC, ameloblastic fibroma, angiosarcoma, and so forth in the routine histopathology resembles malignant picture, and dilemma exists in proper distinction and diagnosis. Hence, knowing the rare granular variants in normal histopathological lesions helps in proper diagnosis and treatment plan.

Immunophenotyping with a comprehensive panel of antibodies, including epithelial, smooth muscle, oncocytic, melanocytic, vascular, and other markers will be useful when granular cell features are diffuse and obscure the underlying lesion. Ultrastructural studies are also extremely useful in excluding lesions that mimic true GCT; identification of well-formed desmosomes, tonofilaments, condensation of thin filaments of actin type, pinocytic vesicles, Weibel-Palade bodies, and melanosomes are all features that indicates another neoplasm rather than true GCT. In the same manner, granular cytoplasmic change in oncocytes, clear cells, rhabdoid cells, and signet ring cells shows different lines of differentiation, and such tumors are better classified on the basis of their more specific features. With regard to prognosis, it appears that granular cell change does not influence the biologic behaviour of smooth muscle tumors. Therefore, granularity in a normal histopathology is a rare but innocuous change in many lesions except few lesions such as cutaneous granular cell angiosarcoma and granular cell ameloblastoma which have shown poor prognosis.

\section{References}

[1] E. P. Sunderland, R. Sunderland, and C. J. Smith, "Granular cells associated with the enamel organ of a developing tooth," Journal of Oral Pathology, vol. 12, no. 1, pp. 1-6, 1983.

[2] K. E. Schoedel, S. Bastacky, and A. Silverman, "An S100 negative granular cell tumor with malignant potential: report of a case," Journal of the American Academy of Dermatology, vol. 39, no. 5, pp. 894-898, 1998.

[3] M. Nasu, M. Takagi, and H. Yamamoto, "Ultrastructural and histochemical studies of granular-cell ameloblastoma," Journal of Oral Pathology, vol. 13, no. 4, pp. 448-456, 1984.

[4] J. A. Regezi, R. J. Zarbo, R. M. Courtney, and J. D. Crissman, "Immunoreactivity of granular cell lesions of skin, mucosa, and jaw," Cancer, vol. 64, no. 7, pp. 1455-1460, 1989.

[5] G. H. Rühl and E. Akuamoa-Boateng, "Granular cells in odontogenic and non-odontogenic tumors," Virchows Archiv A, vol. 415, no. 5, pp. 403-409, 1989.

[6] A. Deshpande, P. Umap, and M. Munshi, "Granular cell ameloblastoma of the jaw: a report of two cases with fine needle aspiration cytology," Acta Cytologica, vol. 44, no. 1, pp. 81-85, 2000.

[7] K. Takahashi, T. Kitajima, M. Lee et al., "Granular cell ameloblastoma of the mandible with metastasis to the third thoracic vertebra. A case report," Clinical Orthopaedics and Related Research, vol. 197, pp. 171-180, 1985.

[8] G. H. Kumamoto and K. Ooya, "Immunohistochemical and ultrastructural investigation of apoptotic cell death in granular cell ameloblastoma," Journal of Oral Pathology and Medicine, vol. 30, no. 4, pp. 245-250, 2001.

[9] R. B. Brannon, R. K. Goode, L. R. Eversole, and R. F. Carr, "The central granular cell odontogenic tumor: report of 5 new cases," Oral Surgery, Oral Medicine, Oral Pathology, Oral Radiology, and Endodontics, vol. 94, no. 5, pp. 614-621, 2002.

[10] Y. Takeda, "Granular cell ameloblastic fibroma, ultrastructure and histogenesis," International Journal of Oral and Maxillofacial Surgery, vol. 15, no. 2, pp. 190-195, 1986.

[11] S. D. Vincent, H. L. Hammond, G. L. Ellis, and J. P. Juhlin, "Central granular cell odontogenic fibroma," Oral Surgery, Oral Medicine, Oral Pathology, vol. 63, no. 6, pp. 715-721, 1987.

[12] A. T. Mesquita, C. R. Santos, R. S. Gomez, J. Jorge, J. E. León, and O. P. de Almeida, "Central granular cell odontogenic tumor: a histopathologic and immunohistochemical study," Annals of Diagnostic Pathology, vol. 13, no. 6, pp. 405-412, 2009. 
[13] M. Altini, J. J. Hille, and A. Buchner, "Plexiform granular cell odontogenic tumor," Oral Surgery, Oral Medicine, Oral Pathology, vol. 61, no. 2, pp. 163-167, 1986.

[14] C. A. Waldron, C. W. Thompson, and W. A. Conner, "Granular-cell ameloblastic fibroma. Report of two cases," Oral Surgery, Oral Medicine, Oral Pathology, vol. 16, no. 10, pp. 1202-1213, 1963.

[15] D. K. White, S. Y. Chen, K. S. Hartman, A. S. Miller, and L. F. Gomez, "Central granular cell tumor of the jaws (the socalled granular-cell ameloblastic fibroma)," Oral Surgery Oral Medicine and Oral Pathology, vol. 45, no. 3, pp. 396-405, 1978.

[16] A. P. Chaudhry, C. T. Hanks, C. Leifer, and E. A. Gargiulo, "Calcifying epithelial odontogenic tumor. A histochemical and ultrastructural study," Cancer, vol. 30, no. 2, pp. 519-529, 1972.

[17] N. A. Abaza, L. Gold, and E. Lally, "Granular cell odontogenic cyst: a unicystic ameloblastoma with late recurrence as follicular ameloblastoma," Journal of Oral and Maxillofacial Surgery, vol. 47, no. 2, pp. 168-175, 1989.

[18] S. Sargenti-Neto, M. T. Brazão-Silva, K. C. do Nascimento Souza et al., "Multicentric granular cell tumor: report of a patient with oral and cutaneous lesions," British Journal of Oral and Maxillofacial Surgery, vol. 47, no. 1, pp. 62-64, 2009.

[19] J. R. Basile and S.-B. Woo, "Polypoid S-100-negative granular cell tumor of the oral cavity: a case report and review of literature," Oral Surgery, Oral Medicine, Oral Pathology, Oral Radiology, and Endodontics, vol. 96, no. 1, pp. 70-76, 2003.

[20] A. Eguia, A. Uribarri, C. G. Escoda, M. A. Crovetto, R. Martínez-Conde, and J. M. Aguirre, "Granular cell tumor: report of 8 intraoral cases," Medicina Oral, Patología Oral y Cirugía Bucal, vol. 11, no. 5, pp. E425-E428, 2006.

[21] D. M. Alessi and M. C. Zimmerman, "Granular cell tumors of the head and neck," Laryngoscope, vol. 98, no. 8, pp. 810-814, 1988.

[22] T. Mentzel, C. Wadden, and C. D. M. Fletcher, "Granular cell change in smooth muscle tumours of skin and soft tissue," Histopathology, vol. 24, no. 3, pp. 223-231, 1994.

[23] M. Lerman and P. D. Freedman, "Nonneural granular cell tumor of the oral cavity: a case report and review of the literature," Oral Surgery, Oral Medicine, Oral Pathology, Oral Radiology and Endodontology, vol. 103, no. 3, pp. 382-384, 2007.

[24] S. E. Thawley and J. H. Ogura, "Granular cell myoblastoma of the head and neck," Southern Medical Journal, vol. 67, no. 9, pp. 1020-1024, 1974.

[25] A. Giménez-Bascuñana and F. M. Piqueras-Pérez, "Granular cell traumatic neuroma of salivary gland," Archives of Pathology and Laboratory Medicine, vol. 125, no. 8, pp. 1000-1001, 2001.

[26] R. Rosso, M. Scelsi, and L. Carnevali, "Granular cell traumatic neuroma: a lesion occurring in mastectomy scars," Archives of Pathology and Laboratory Medicine, vol. 124, no. 5, pp. 709711, 2000.

[27] J. Dash, P. Sahoo, and S. Das, "Congenital granular cell lesion "congenital epulis"- report of a case," Journal of the Indian Society of Pedodontics and Preventive Dentistry, vol. 22, no. 2, pp. 63-67, 2004.

[28] A. M. Loyola, A. F. Gatti, D. S. Pinto Jr, and R. A. Mesquita, "Alveolar and extra-alveolar granular cell lesions of the newborn: report of case and review of literature," Oral Surgery, Oral Medicine, Oral Pathology, Oral Radiology, and Endodontics, vol. 84, no. 6, pp. 668-671, 1997.
[29] I. Bhattacharyya, D.-J. Summerlin, D. M. Cohen, G. L. Ellis, J. B. Bavitz, and L. L. Gillham, "Granular cell leiomyoma of the oral cavity," Oral Surgery, Oral Medicine, Oral Pathology, Oral Radiology and Endodontology, vol. 102, no. 3, pp. 353359, 2006.

[30] Y. Dobashi, K. Iwabuchi, J. Nakahata, K. Yanagimoto, and T. Kameya, "Combined clear and granular cell leiomyoma of soft tissue: evidence of transformation to a histiocytic phenotype," Histopathology, vol. 34, no. 6, pp. 526-531, 1999.

[31] I. G. Koutlas, S. E. Pambuccian, J. Jessurun, J. C. Manivel, and R. Gopalakrishnan, "Perivascular epithelioid cell tumor of the oral mucosa," Archives of Pathology and Laboratory Medicine, vol. 129, no. 5, pp. 690-693, 2005.

[32] J. Kanitakis and B. Chouvet, "Granular-cell basal cell carcinoma of the skin," European Journal of Dermatology, vol. 15, no. 4, pp. 301-303, 2005.

[33] R. E. Mrak and G. F. Baker, "Granular cell basal cell carcinoma," Journal of Cutaneous Pathology, vol. 14, no. 1, pp. 37-42, 1987.

[34] M. Ladanyi, C. R. Antonescu, M. Drobnjak et al., "The precrystalline cytoplasmic granules of alveolar soft part sarcoma contain monocarboxylate transporter 1 and CD147," American Journal of Pathology, vol. 160, no. 4, pp. 1215-1221, 2002.

[35] A. L. Folpe and A. T. Deyrup, "Alveolar soft-part sarcoma: a review and update," Journal of Clinical Pathology, vol. 59, no. 11, pp. 1127-1132, 2006.

[36] T. O. Nielsen, G. Séjean, and R. M. Onerheim, "Paraganglioma of the tongue," Archives of Pathology and Laboratory Medicine, vol. 124, no. 6, pp. 877-879, 2000.

[37] H. I. Lee, J. W. Lee, T. Y. Han et al., "A case of dermatofibroma of the upper lip," Annals of Dermatology, vol. 22, no. 3, pp. 333-336, 2010.

[38] F. Aloi, D. Albertazzi, and M. Pippione, "Dermatofibroma with granular cells: a report of two cases," Dermatology, vol. 199, no. 1, pp. 54-56, 1999.

[39] T. R. Helliwel, M. C. Sissons, P. J. Stoney, and M. T. Ashworth, "Immunochemistry and electron microscopy of head and neck rhabdomyoma," Journal of Clinical Pathology, vol. 41, no. 10, pp. 1058-1063, 1988.

[40] P. E. LeBoit, R. J. Barr, S. Burall, J. S. Metcalf, T. S. B. Yen, and M. R. Wick, "Primitive polypoid granular-cell tumor and other cutaneous granular-cell neoplasms of apparent nonneural origin," American Journal of Surgical Pathology, vol. 15, no. 1, pp. 48-58, 1991.

[41] R. Mirchandani, J. J. Sciubba, and R. Mir, "Granular cell lesions of the jaws and oral cavity: a clinicopathologic, immunohistochemical, and ultrastructural study," Journal of Oral and Maxillofacial Surgery, vol. 47, no. 12, pp. 1248-1255, 1989.

[42] http://moon.ouhsc.edu/kfung/jty1/Com08/Com807-1-Diss .htm.

[43] A. C. Stîngă, A. S. Stîngă, C. Simionescu, C. Mărgăritescu, and M. Cruce, "Histopathological study of renal cell carcinoma," Current Health Sciences Journal, vol. 35, no. 1, pp. 52-57, 2009.

[44] L. D. Thompson, B. M. Wenig, and G. L. Ellis, "Oncocytomas of the submandibular gland: a series of 22 cases and a review of the literature," Cancer, vol. 78, no. 11, pp. 2281-2287, 1996.

[45] M. Shintaku and T. Honda, "Identification of oncocytic lesions of salivary glands by anti- mitochondrial immunohistochemistry," Histopathology, vol. 31, no. 5, pp. 408-411, 1997.

[46] G. Giordano, M. Gabrielli, L. Gnetti, and T. Ferri, "Oncocytic carcinoma of parotid gland: a case report with clinical, 
immunohistochemical and ultrastructural features," World Journal of Surgical Oncology, vol. 4, article 54, 2006.

[47] L. Corcione, G. Giordano, L. Gnetti, A. Multinu, and S. Ferrari, "Oncocytic mucoepidermoid carcinoma of a submandibular gland: a case report and review of the literature," International Journal of Oral and Maxillofacial Surgery, vol. 36, no. 6, pp. 560-563, 2007.

[48] T. D. Daley, G. P. Wysocki, M. S. Smout, and R. P. Slinger, "Short communication \& case report: epithelial-myoepithelial carcinoma of salivary glands," Oral Surgery Oral Medicine and Oral Pathology, vol. 57, no. 5, pp. 512-519, 1984.

[49] K. Mardi and J. Sharma, "Oncocytic carcinoma: a rare malignancy of the parotid gland," The Internet Journal of Pathology, vol. 8, no. 2, 2009.

[50] M. J. Franzblau, M. Manwaring, C. Plumhof, M. B. Listrom, and W. H. C. Burgdorf, "Metastatic breast carcinoma mimicking granular cell tumor," Journal of Cutaneous Pathology, vol. 16, no. 4, pp. 218-221, 1989.

[51] S. Rai, R. Naik, M. R. Pai, and A. Gupta, "Metastatic hepatocellular carcinoma presenting as a gingival mass," Indian Journal of Pathology and Microbiology, vol. 52, no. 3, pp. 446-447, 2009.

[52] B. Zelger, R. Cerio, G. Orchard, and E. Wilson-Jones, "Juvenile and adult xanthogranuloma: a histological and immunohistochemical comparison," American Journal of Surgical Pathology, vol. 18, no. 2, pp. 126-135, 1994.

[53] A. Consolaro, E. Sant'Ana, M. A. Lawall, M. F. Consolaro, and C. E. Bacchi, "Gingival juvenile xanthogranuloma in an adult patient: case report with immunohistochemical analysis and literature review," Oral Surgery, Oral Medicine, Oral Pathology, Oral Radiology and Endodontology, vol. 107, no. 2, pp. 246-252, 2009.

[54] M. G. Hitchcock, M. A. Hurt, and D. J. Santa Cruz, "Cutaneous granular cell angiosarcoma," Journal of Cutaneous Pathology, vol. 21, no. 3, pp. 256-262, 1994. 


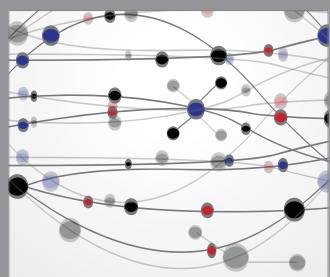

The Scientific World Journal
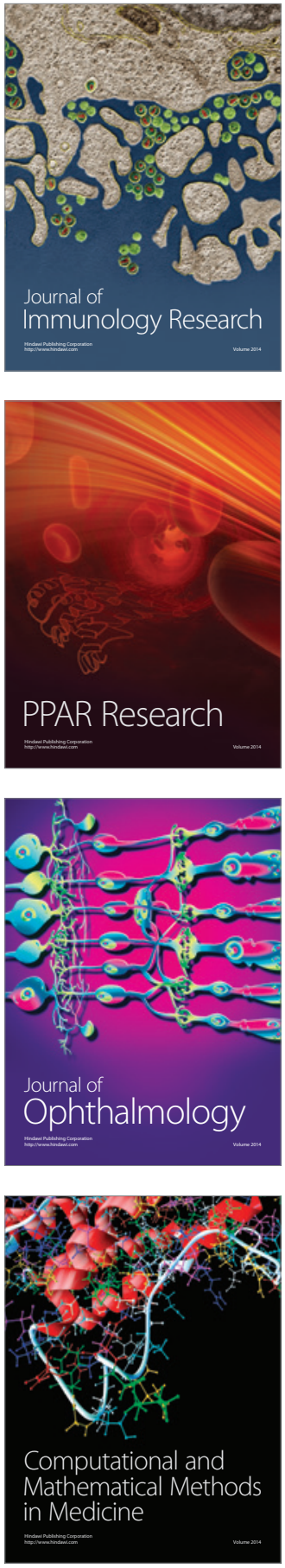

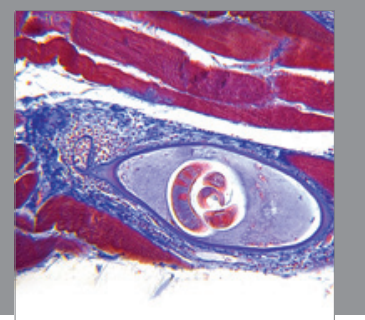

Gastroenterology

Research and Practice
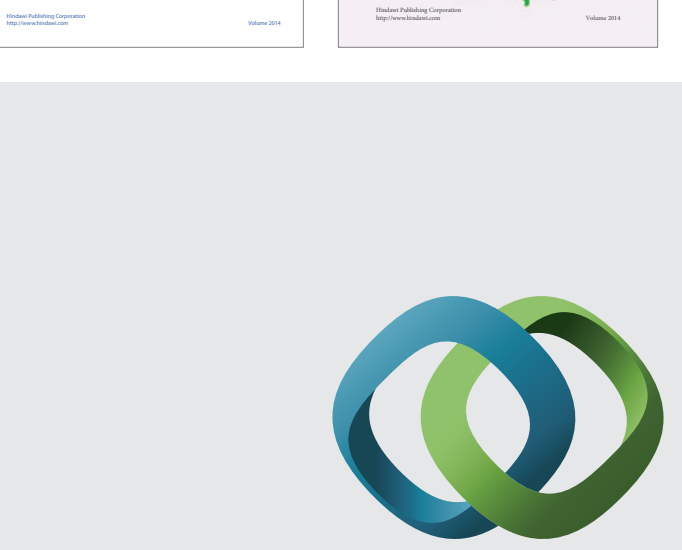

\section{Hindawi}

Submit your manuscripts at

http://www.hindawi.com
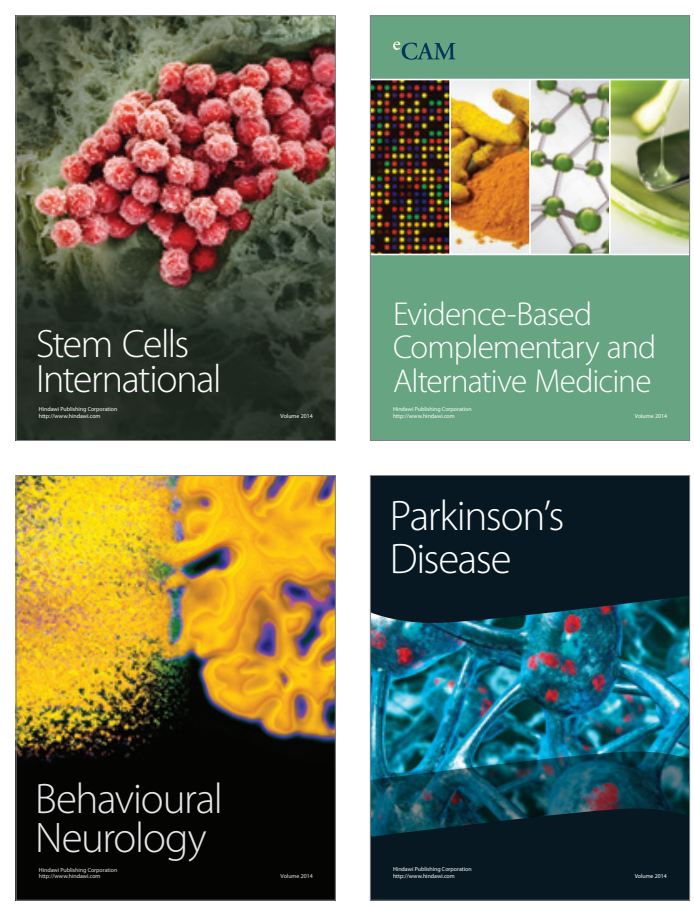

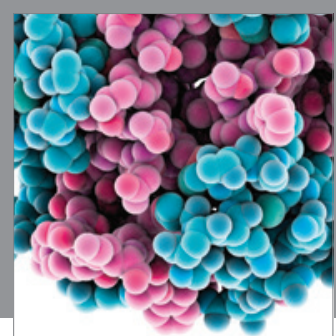

Journal of
Diabetes Research

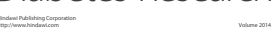

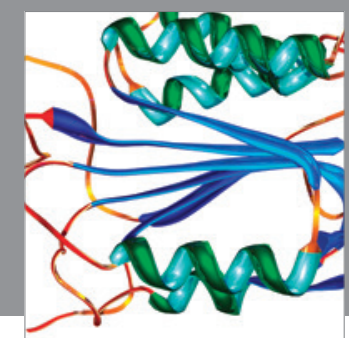

Disease Markers
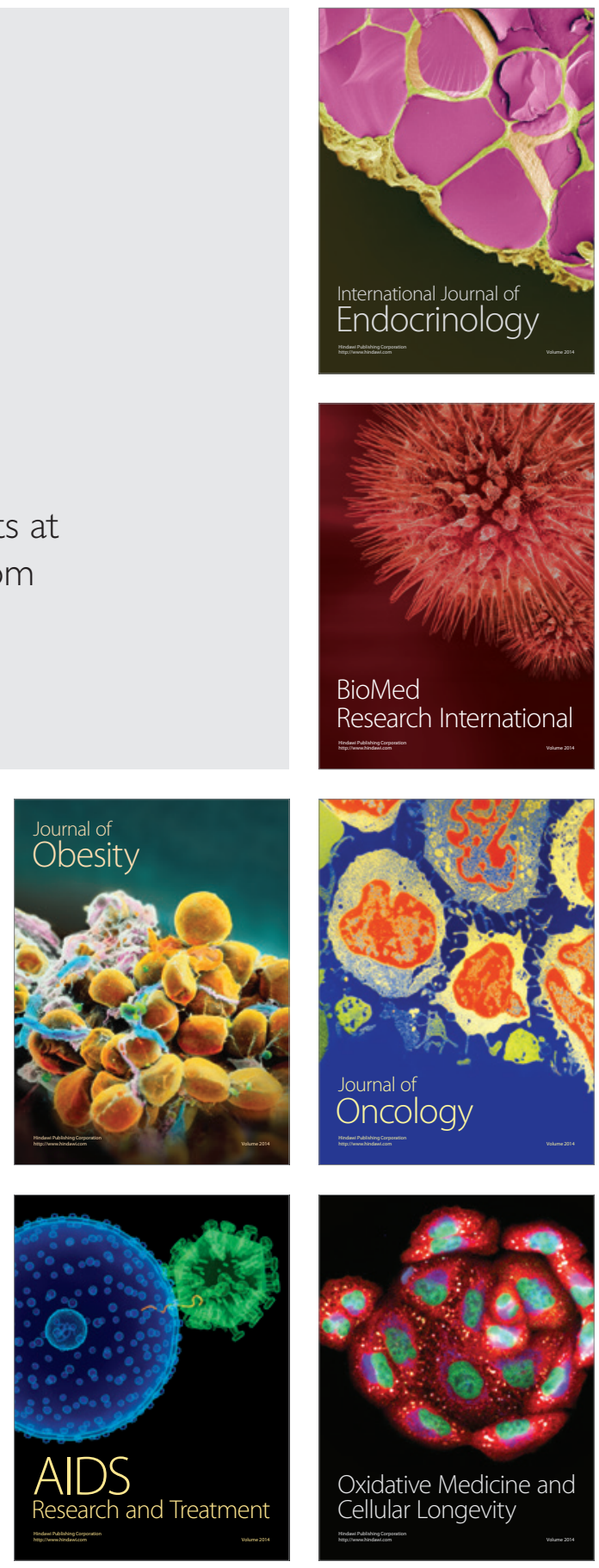